\title{
A Model of High-Frequency Self-Mixing in Double-Barrier Rectifier
}

\author{
Fabrizio Palma ${ }^{1}$ (iD $\cdot$ R. Rao ${ }^{1}$
}

Received: 1 September 2017 / Accepted: 22 February 2018

(C) Springer Science+Business Media, LLC, part of Springer Nature 2018

\begin{abstract}
In this paper, a new model of the frequency dependence of the double-barrier $\mathrm{THz}$ rectifier is presented. The new structure is of interest because it can be realized by CMOS image sensor technology. Its application in a complex field such as that of $\mathrm{THz}$ receivers requires the availability of an analytical model, which is reliable and able to highlight the dependence on the parameters of the physical structure. The model is based on the hydrodynamic semiconductor equations, solved in the small signal approximation. The model depicts the mechanisms of the $\mathrm{THz}$ modulation of the charge in the depleted regions of the doublebarrier device and explains the self-mixing process, the frequency dependence, and the detection capability of the structure. The model thus substantially improves the analytical models of the $\mathrm{THz}$ rectification available in literature, mainly based on lamped equivalent circuits.
\end{abstract}

Keywords THz sensor $\cdot$ Double-barrier rectifier $\cdot$ Pinned photodiode $\cdot$ CMOS

\section{Introduction}

The detection of high-frequency electromagnetic radiation by integrated circuit, in particular of terahertz radiation $(\mathrm{THz})$, with the goal to achieve resolute images, eventually colored by the interaction with material chemical bonding, represent a very challenging task which is pushing great experimental and theoretical activities. The radiations spectrum of interest practically covers all the gap between the microwaves and the infrared regions. The strong penetrability, the sensitivity to chemical bonds, and the high resolution make the $\mathrm{THz}$ range an attractive tool for different applications, as medical imaging and security check.

Since the THz radiation is non-ionizing and the associated power is low, it is considered safe. Nevertheless, $\mathrm{THz}$ wavelength is able to deeper scan the material under investigation.

Fabrizio Palma

fabrizio.palma@uniroma1.it

1 Rome Università La Sapienza, Rome, Italy 
The combination between the safe and penetrating characteristics can be the winning qualities in different applications, such as medical imaging, security/surveillance imaging, and spectroscopic applications.

Recently, a strong effort was spent in order to achieve THz sensors by using the standard, low-cost, CMOS technology which, in particular, is characterized by a strong reproducibility on a large area. This characteristic allows to achieve arrays of detectors, useful to have detector panels for a large area approach.

Until now, the high-frequency detection, based on CMOS technology [1-4], was achieved using a mechanism described by the plasma wave detection theory [5]. When a high-frequency signal is applied between gate and source electrodes of a MOSFET, a $2 \mathrm{D}$ electron gas (located in the inversion layer) converts $\mathrm{THz}$ radiation into a DC voltage. In order to increase the detector responsivity, it was demonstrated that a strong downscaling of the gate length is necessary [6]. Nevertheless, also for ultra-scaled technology nodes, the device parasitic capacitance significantly influences the detector responsivity [7].

We recently proposed a new structure of high-frequency radiation detector [8], based on the CMOS image sensor technology. The rectifying device is not a MOSFET, substituted by a CMOS compatible new device, the double-barrier structure, while the already well-established pixel electronics is used for the charge sensing and amplification after the detection. The new double-barrier structure is based on the combination of the $\mathrm{n} / \mathrm{p}^{+}$barrier and a $\mathrm{p}^{+} / \mathrm{metal}$ Schottky barrier. The double-barrier is mandatory to preserve the storage well structure of the pinned photodiode [9], where the rectified charge has to be collected. The collected charge is then read by the standard CMOS image sensor electronics, after the integration time.

The proposed structure is very effective in rectification of radiofrequency signal, as reported in [8]. The asymmetry between the two barriers is the basis of the rectification capability. The reported Technology Computer Aided Design (TCAD) simulations of the double-barrier structure indicate that the detection capability is preserved up to very high-frequency range, with a cutoff frequency of at least of $120 \mathrm{GHz}$. This property allows an effective application in the THz range. This behavior is confirmed by comparison with the Schottky diode frequency multiplier [10], where measurements and TCAD simulations show a smooth drop of the conversion efficiency, until $840 \mathrm{GHz}$, followed by a drastic reduction above $1.4 \mathrm{THz}$.

The application of the new structure in a complex field such as that of $\mathrm{THz}$ receivers requires the availability of an analytical model, which is reliable and able to highlight the dependence on the parameters of the physical structure.

In contrast with the evidence of rectifying capabilities up to very high frequency, the analytical models of the $\mathrm{THz}$ rectification available in literature, mainly based on lamped equivalent circuits, show in general $1 / \omega^{2}$ dependence. For instance, in [11] an equivalent circuit, with parameters obtained from low-frequency measurements, is used for the development of an extended model of the Schottky barrier, while in [12] contact skin effect, and bulkspreading impedance was adopted. Nevertheless, a simply lumped barrier impedance was used. Han et al. [13] related either the noise or the responsivity to the DC first and second derivative of the Schottky diode I-V characteristics.

In contrast with these approaches, the Monte Carlo simulations [14] demonstrate how in the Schottky diodes, the capacitance/voltage $(\mathrm{C}-\mathrm{V})$ nonlinearity dominate with respect to the current/voltage (I-V) nonlinearity in the range $0.2-5 \mathrm{THz} . C-V$ nonlinearities depend on the charge inside the depletion layer, which cannot be described by the usual abrupt depletion layer approximation which, in the small signal approximation, would led to a constant equivalent capacitance. 
In this paper, we develop a new model of the self-mixing process in the double-barrier device, a model that in principle can be extended also to different structures, e.g., the single barrier.

The model is based on the hydrodynamic semiconductor equations, solved in the small signal approximation. The detector is assumed in the zero bias condition, as expected in a detector, in order to reduce the junction noise. The model depicts the mechanisms of the $\mathrm{THz}$ modulation of the charge in the depleted regions of the structure. As a result, we obtain the frequency dependence of the detection capability of the double-barrier detector.

In the model, the frequency dependence of the rectification responsivity is not related to the damping effect of the parasitic capacitances, but to the nonlinear effect of the carrier dynamic within the depletion layer, the self-mixing process.

In our approach, thanks to the hydrodynamic semiconductor equations, we are able to highlight different contributions coming from different portions of the two barriers structure. Our approach connects both to the model of resonant-mixing detection in two dimensional electronic fluids [5] and to the self-mixing model in semiconductor layered structures [15].

\section{The Analytical Model}

A sketch of the double-barrier structure is reported in Fig. 1. The origin is placed at the left border of the depletion region of the n-type layer. At the same edge, it is placed an ohmic contact (cathode). The $\mathrm{n} / \mathrm{p}^{+}$junction is assumed at $x=w_{n}$, where $w_{n}$ is the depletion region depth in the n-type silicon side, in the "abrupt edge" depletion approximation. The $\mathrm{p}^{+}$region has thickness $D$ and is assumed as completely depleted. The $\mathrm{p}^{+}$region and the metal form a Schottky barrier, located at $x=W=w_{n}+D$. The metal forms the anode contact.

We first define the steady-state conditions of the structure. Due to the charge balance, we can impose the following balance equation:

$$
\frac{q}{\epsilon}\left(w_{n} N_{D}-D N_{A}\right)=E_{2}
$$

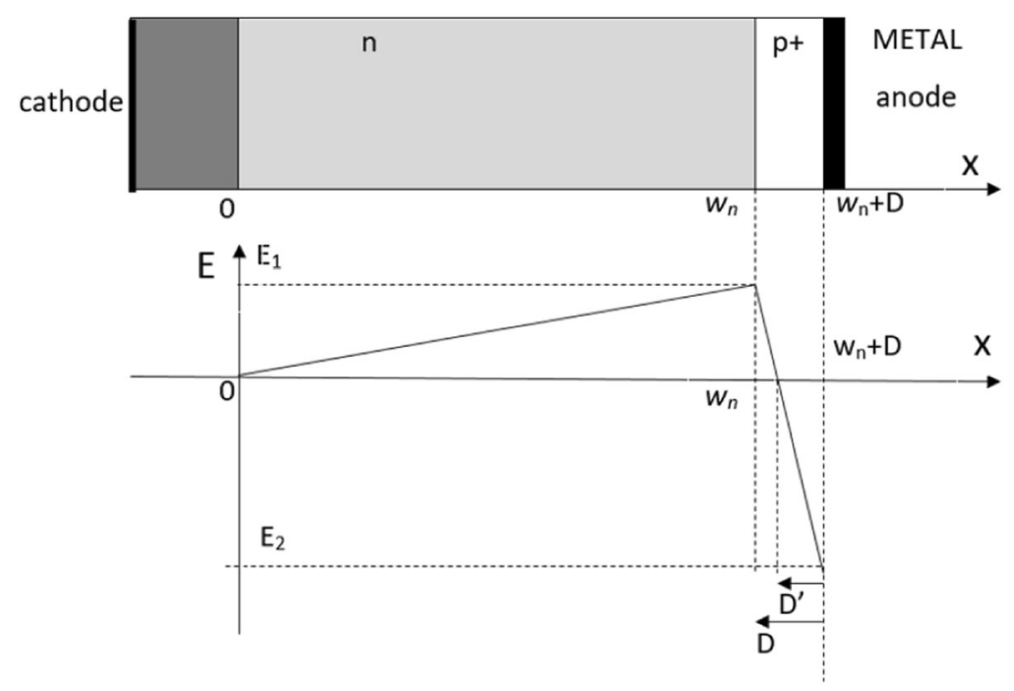

Fig. 1 The semiconductor structure and the sketch of the electric field distribution 
where $N_{D}$ is the density of donors in the n region, $N_{A}$ is density of acceptors in the $\mathrm{p}^{+}$region, $q$ is the absolute value of the electron charge, $\epsilon$ the dielectric constant in the semiconductor, and $E_{2}$ is the electric field at the $\mathrm{p}^{+} /$metal interface due to charge collected in the metal layer.

The built-in potential across the structure is due to the difference between the Fermi level in the $\mathrm{n}$ layer and the metal work function. Assuming a metal work function of $F_{M}=4.33 \mathrm{eV}$ (titanium), we can write the built-in potential as reported below:

$$
V_{\mathrm{BIN}}=\left(q \chi_{n}+\frac{E_{G}}{2}-\frac{k_{B} T}{q} \log \left(\frac{N_{D}}{n_{i}}\right)\right)-F_{M}
$$

where $q \chi_{n}$ is the electron affinity of the n-doped silicon, $k_{B}$ the Boltzman constant, $T$ the absolute temperature, $E_{G}$ the semiconductor gap, and $n_{i}$ the intrinsic carrier concentration. The steady-state electric field in the structure can be analytically expressed as follows:

$$
\begin{gathered}
E_{0}(x)=\frac{q}{\epsilon} x N_{D} \text { for } x<w_{n} \\
E_{0}(x)=E_{1}+\frac{q}{\epsilon} x N_{A}\left(x-w_{n}\right) \text { for } x>w_{n}
\end{gathered}
$$

where $E_{1}=\frac{q}{\epsilon} w_{n} N_{D}$. We can integrate Eqs. (3) and (4) in order to obtain the potential drop across the structure and impose it to be equal to the built-in voltage:

$$
\int_{0}^{w_{n}} \frac{q}{\epsilon} x N_{D} d x+\int_{0}^{D} E_{1}+\frac{\left(E_{2}-E_{1}\right)}{D} x^{\prime} d x^{\prime}=V_{\mathrm{BIN}}
$$

Using Eqs. (1) and (5), the depletion region width, $w_{n}$, and the value of $E_{2}$ can be calculated.

Assuming the thermal equilibrium condition for the carriers inside the structure, due to the zero bias condition, and zero potential at $x=0$, the electron density is given by the following:

$$
n_{0}(x)=n_{n 0} e^{\frac{\phi(x)}{k_{B} T}}
$$

where $\phi(x)$ is the potential distribution inside the structure, and $n_{n 0}$ is the equilibrium electron density at the cathode contact. Figure 2 reports the behavior of $n_{0}$ inside the structure, assuming the value of the numerical example presented in section III.

Fig. 2 Comparison of the two homogeneous terms in Eq. (14), from solutions $\hat{n}_{C}$ (continuous) and $\hat{n}_{A}$ (dashed), at the frequencies of $320 \mathrm{GHz}$ (blue), $1 \mathrm{THz}$ (green), and $3.2 \mathrm{THz}$ (red), respectively

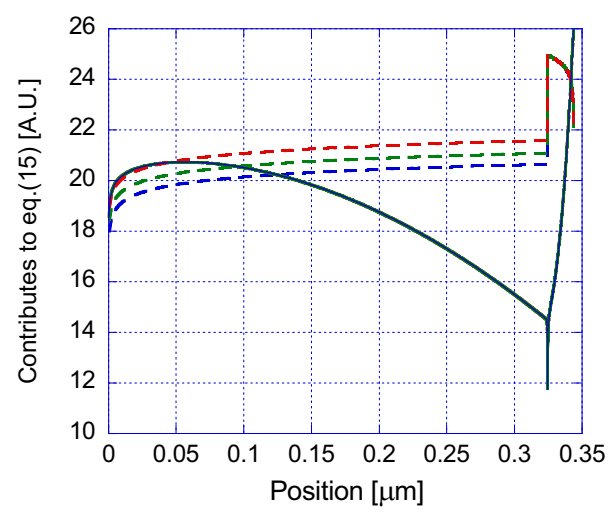


An equivalent expression can be obtained assuming the zero potential at $x=W$ :

$$
n_{0}(x)=n_{n 0} e^{V_{\mathrm{BIN}}} e^{\frac{\phi^{\prime}(x)}{q}}=n_{n M} e^{\frac{\phi^{\prime}(x)}{q}}
$$

where $\phi^{\prime}(x)=\phi(x)-V_{B I N} . n_{n M}$ is the electron density at $x=W$, imposed by the metal work function.

In order to describe the variation of the physical quantities in the structure, we introduce the hydrodynamic semiconductor equation written as follows:

$$
\begin{gathered}
\frac{\partial \phi}{\partial x}=-E \\
\frac{\partial E}{\partial x}=\frac{q}{\varepsilon} n \\
\frac{d n}{d t}=-\frac{\partial n v}{\partial x}+D_{n} \frac{\partial^{2} n}{\partial^{2} x} \\
\frac{\partial v}{\partial t}=v \frac{\partial v}{\partial x}+\frac{q}{m} E-\frac{v}{\tau}
\end{gathered}
$$

where $n$ is the total electrons density (steady-state plus variations), and $v$ is the total electrons velocity. In this set of equations, we neglect the transport of holes, assuming them as minority even in the depleted $\mathrm{p}^{+}$region.

The delay due to the kinetic of electron, accelerated by the electric field, is described by the Euler Eq. (11) limited by the effect of electron collision, where $\tau$ is the collision time.

In order to achieve a correct solution within the depletion layer of the junctions, we take into account both the contributions due to transport and diffusion. The effect of the diffusion is assumed instantaneous, thus considering the phonon frequency higher than the frequency of applied signal. It gives rise to a limitation of the electron density variation at the lower frequency. Its contribution starts decreasing above a frequency of $10^{11} \mathrm{~Hz}, \mathrm{n}$ practice limiting the general decrease of electron variation. Assuming only the transport term, combining the dependence induced by the Euler equation with the continuity equation would result on a frequency dependence proportional to $1 / \omega^{2}$, while, including the diffusion contribution, the model obtained leads to a sub-linear dependence at low frequencies.

We consider that a sinusoidal radiofrequency voltage drop, $V_{R F}(t)=\hat{v}_{R F} e^{-j \varpi t}$, is applied to the structure (positive sign assumed at the cathode). In the sharp edge approximation, we can assume that the maximum charge variation is located at the edges of the structure, at the edge of the depletion layer in the zone $\mathrm{n}, x=0$, and in the metal, $x=W$. With this assumption, the first-order variations of the electric field inside the structure do not depend on the position. Therefore, we can write $\hat{E}(x)=\hat{v}_{R F} / w$.

With the assumption of a constant electric field, also the amplitude of the first-order electron velocity variation can be assumed constant along the depletion region. Moreover, thanks to this assumption, we can neglect the nonlinear term in Eq. (11). 
Assuming the small signal approximation, the first-order variation of the electron density has a sinusoidal variations in time: $n(x, t)=n_{0}(x)+\hat{n}(x) e^{-j \varpi t}$, and variations of the electron drift velocity are constant in space and sinusoidal in time: $v(t)=v_{0}(x)+\hat{v} e^{-j \varpi t}$. The semiconductor equations for the variations can be simplified as follows:

$$
\begin{gathered}
\hat{E} \cong \hat{v}_{R F} / W \\
\hat{v}=\frac{\frac{q}{m} \hat{E}}{j \omega+\frac{1}{\tau}} \\
j \varpi \hat{n}=-\left[\hat{v} \frac{\partial n_{0}}{\partial x}+\hat{n} \frac{\partial v_{0}}{\partial x}+v_{0} \frac{\partial \hat{n}}{\partial x}\right]+D_{n} \frac{\partial^{2} \hat{n}}{\partial^{2} x}
\end{gathered}
$$

In the last equation, Eq. (14), a term $n_{0} \frac{\partial \hat{v}}{\partial x}$ is neglected, due to the assumption that velocity variations are constant in space within the depletion layer. The term $\partial n_{0} / \partial x$, in the following referred as $n_{1}$, can be analytically evaluated using the steady-state distribution of electrons, Eqs. (6) and (7), and the distribution of the electric field ((3) and (4)). Therefore, assuming the spatial constancy of the variations of electric field and velocity, substituting Eqs. (12) and (13) into the differential Eq. (14), we find that the term $v \frac{\partial n_{0}}{\partial x}$ represents a non-homogeneous term.

We also assume the presence of a DC term of the velocity into the depletion layer: $v_{0}(x)=$ $-\mu_{n} E_{0}(x)$, where the steady-state electric field in the different regions is given by expressions (3) and (4). We obtain for Eq. (14):

$$
\left(j \varpi-\frac{q}{\epsilon} \mu_{n} N_{J}\right) \hat{n}-\mu_{n} E_{0} \frac{\partial \hat{n}}{\partial x}-D_{n} \frac{\partial^{2} \hat{n}}{\partial^{2} x}=-\hat{v} n_{1}
$$

where $N_{J}=N_{D}$ is in the region $x<w_{n}$, and $N_{J}=N_{A}$ is in the region $w-D>x>w$. In order to overcome difficulties in obtaining the exact solution of Eq. (15), we adopt an "asymptotic" approach. In this approach, we assume that the effect of each one of the three homogeneous terms can be dominant or negligible, depending on the position inside the junction and on the frequency value. We thus evaluate three distinct approximated solutions, respectively, $\hat{n}_{A}(x)$, $\hat{n}_{B}(x)$, and $\hat{n}_{C}(x)$, in which the effect of only one of the three homogeneous terms is considered dominant. The three asymptotic solutions are given by the following:

$$
\begin{gathered}
\text { considering the first term : } \hat{n}_{A}=\frac{\hat{v} n_{1}(\mathrm{x})}{\frac{q}{\epsilon} \mu_{n} N_{D-j \varpi}} \\
\text { considering the second term }: \frac{\partial \hat{n}_{B}}{\partial x}=\frac{\hat{v}}{\mu_{n} E_{0}} \frac{\partial n_{0}}{\partial x}
\end{gathered}
$$

with $\hat{n}_{B}=\frac{\hat{v} n_{0}}{\mu_{n} E}+$ constant and:

$$
\text { considering the third term : } D_{n} \frac{\partial^{2} \hat{n}_{C}}{\partial^{2} x}=\hat{v} n_{1}(x)
$$

Solution $\hat{n}_{A}$ has the same spatial distribution of $n_{1}(x)$, and a strong frequency dependence. At a very high frequency, the term results in quadrature with $\hat{v}$. Solution $\hat{n}_{B}$ has the same spatial 
distribution of $n_{0}(x)$, and it is in phase with $\hat{v}$. We note that $\hat{n}_{B}$ cannot represent the solution at $x=0$, since there, $E_{0}$ is null. Regarding solution $\hat{n}_{C}$, in the region $x<w_{n}$, with negative slope of the electron density, from the known spatial distribution of $n_{1}(x)$, Eq. (18) can be integrated:

$$
\hat{n}_{C}(x)=\frac{\hat{v}}{D_{n}} \int_{0}^{\mathrm{x}} \int_{0}^{\mathrm{x}^{\prime}} n_{1} d x^{\prime \prime} d x^{\prime}+n(0)=\frac{\hat{v}}{D_{n}} \int_{0}^{\mathrm{x}} n_{0} d x^{\prime}
$$

where the variation of electrons density at the edge of the depletion layer, $n(0)$, is assumed to be null. Substituting Eqs. (3), (4), (6), and (8) into the Eq. (19), we obtain (for $0<x<w_{n}$ ):

$$
\hat{n}_{C}(x)=\frac{\hat{v}}{D_{n}} \int_{0}^{x} n_{n 0} \mathrm{e}^{-\frac{1 E_{1}}{2} x^{\prime 2}} d x^{\prime}=\frac{\pi}{2} \frac{\hat{v}}{D_{n}} n_{n 0} \frac{1}{\xi} \operatorname{erf}(\xi x)
$$

where $\xi=\sqrt{\frac{E_{1}}{2 w_{n} v_{T}}}$. Below the collision frequency, this solution is not depending on the frequency. The only frequency dependence is given from the variation of the validity region.

Solution (20) of $\hat{n}_{C}$ is valid only in a region with negative slope of the electrons density. In open-circuit condition, we assume that the whole amount of electrons, varying following Eq. (20), is fed by the reservoir of electrons of the non-depleted $\mathrm{n}$ region.

In the point $D^{\prime}$ (see Fig. 1), the electric field is null. In the region $D^{\prime}<x<\mathrm{W}$, with positive slope of the electrons density, we assume that the total amount of electrons varying into this portion of the structure is furnished by the metal at $x=W$. For this solution, we impose as boundary condition $n(W)=0$, and, for a clearer formulation, we change the variable, $z=W-x$. Integrating in this zone is follows:

$$
\hat{n}_{C}^{\prime}(z)=n(W)+\frac{\hat{v}}{D_{n}} \int_{0}^{z} \int_{0}^{z^{\prime}} n_{1} d z^{\prime \prime} d z^{\prime}=\frac{\hat{v}}{D_{n}} \int_{0}^{z} n_{0}\left(z^{\prime}\right) d z^{\prime}
$$

Assuming for $n_{0}(x)$, in expression (7), we can write $\left(D^{\prime}<z<0\right)$ :

$$
\begin{gathered}
\hat{n}_{C}^{\prime}(z)=-\frac{\hat{v}}{D_{n}} \int_{0}^{z} n_{n M} \mathrm{e}^{-\frac{\left|E_{2}\right|}{2 D^{\prime} V_{T}}\left(2 D^{\prime} z^{\prime}-z^{\prime 2}\right)} d z^{\prime}= \\
=\frac{\pi}{2} \frac{\hat{v}}{D_{n}} n_{n M} e^{-\frac{\left|E_{2}\right| D^{\prime}}{2 V_{\mathrm{T}}}} \frac{1}{\zeta}\left\{\operatorname{erf}\left(\zeta \mathrm{D}^{\prime}\right)-\operatorname{erf}\left[\zeta\left(D^{\prime}-z\right)\right]\right\}
\end{gathered}
$$

where $\zeta=\sqrt{\frac{\left|E_{2}\right|}{2 D^{\prime} v_{T}}}$. An additional function should be evaluated, for the interval between $w_{n}$ and $W-D^{\prime}$. Nevertheless, since the dimension of this area is very small, and since the diffusion term became negligible in this zone due to the low carrier concentration, we can avoid this calculation.

Each asymptotic solution will be a valid approximation only in a given portions of the structure; this portions depend in general on the operating frequency. The choice between the three solutions, $\hat{n}_{A}, \hat{n}_{B}$, and $\hat{n}_{C}$, cannot be done by a direct comparison of the three functions, since they contribute to Eq. (14) according to different operators. We must then compare the contributions after the operator's effect. In particular, if we assume as valid solution $\hat{n}_{C}$, Eq. (18) allows to evaluate directly the effect of the 
operator tem as $T_{C}=\hat{v} n_{1}(x)$. Evaluating also the other two operators for this same solution $\hat{n}_{C}$, we obtain the following:

$$
\begin{gathered}
T_{A}=\left(j \varpi-\frac{q}{\epsilon} \mu_{n} N_{J}\right) \hat{n}_{C}(x) \\
T_{B}=-\mu_{n} E_{0} \frac{\partial \hat{n}_{C}}{\partial x}=-\mu_{n} E_{0} \frac{\hat{v}}{D_{n}} n_{0}
\end{gathered}
$$

where in Eq. (24), Eq. (19) or (21) depending on the zone, has been used. Numerical evaluation of the three terms shows that $T_{B}$ is negligible everywhere at the frequency of interest. Figure 2 reports in arbitrary units the amplitude of the two main terms, at the frequencies of $320 \mathrm{GHz}$ and 1 and 3.2 THz, respectively. We can accept solution $\hat{n}_{A}$ only in the inner zones, in which its contribution, according to the correspondent operator, is the greatest. Since the term $T_{A}$ has a direct dependence on the frequency, while $T_{C}$ has only the frequency dependence of $v$, the validity region of the diffusion solution $\hat{n}_{A}$ reduces increasing the frequency.

In Fig. 3 a, b, c, the distribution of real and imaginary part of the first-order variations of electron density are reported, at the frequencies of $320 \mathrm{GHz}$ and 1 and $3.2 \mathrm{THz}$, respectively, as obtained from the two solutions $\hat{n}_{A}$ and $\hat{n}_{C}$. We note that the ratios between the real and the imaginary parts inverts in the two solutions. In particular, following Eq. (16), we find that at the highest frequencies, the solution $\hat{n}_{A}$ reduces in module and tends to become in quadrature with the velocity.

On the contrary, solution $\hat{n}_{C}$ remains in phase, but the area of applicability reduces. The sharp discontinuities are justified by asymptotic approach to the solution.

The electron density variation contributes to the nonlinear second term of Eq. (10), giving rise to a DC self-mixing term, $J_{\mathrm{DC}}=-q\langle n v\rangle$, as the time average of the electron flux. We note that only the product between first-order terms in phase (we neglect higher order terms) gives a DC term not null. Thus, from definitions (16), it results that the contribution of solution $\hat{n}_{A}$, $J_{\mathrm{DC}_{-} \mathrm{A}}=\left\langle q n_{A} v\right\rangle$ is null at high frequency, when it becomes in quadrature with the velocity. Solution $\hat{n}_{C}$, always in phase, remains the only contribution to the DC self-mixing; nevertheless, its validity zone is canceled at high frequency, so that there is a sharp decrease of the overall self-mixing effect at high frequency. Figure 4 reports the distribution of the DC selfmixing current generated in the depletion layer, for $0<x<w_{n}$, at $320 \mathrm{GHz}$ and 1 and $3.2 \mathrm{THz}$, respectively.
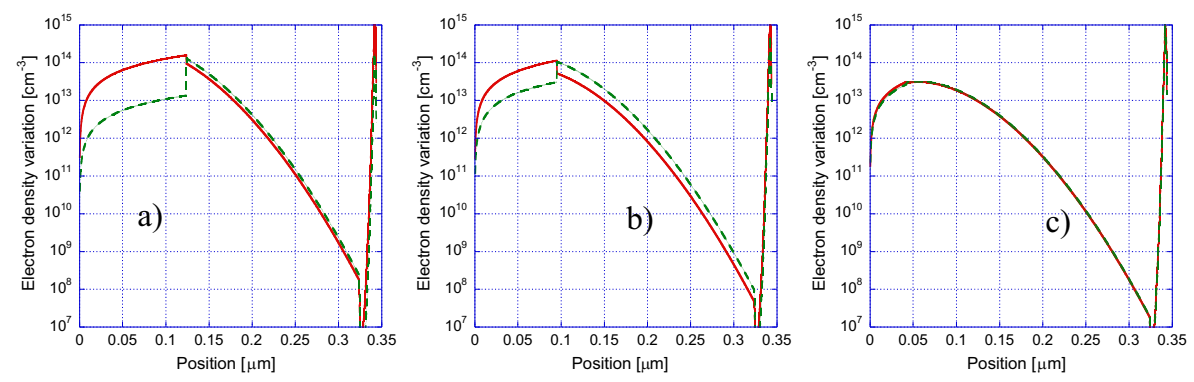

Fig. 3 a-c $\log$ of the distribution of real and imaginary parts of the first-order variations of electron density, as obtained from the two solutions $\hat{n}_{A}$ (green) and $\hat{n}_{C}$ (red), respectively, at $320 \mathrm{GHz}$ and 1 and $3.2 \mathrm{THz}$, respectively 
Fig. 4 Distribution of the DC selfmixing current generated in the depletion layer, at the frequencies of $320 \mathrm{GHz}$ (blue), $1 \mathrm{THz}$ (green), and $3.2 \mathrm{THz}$ (red), respectively

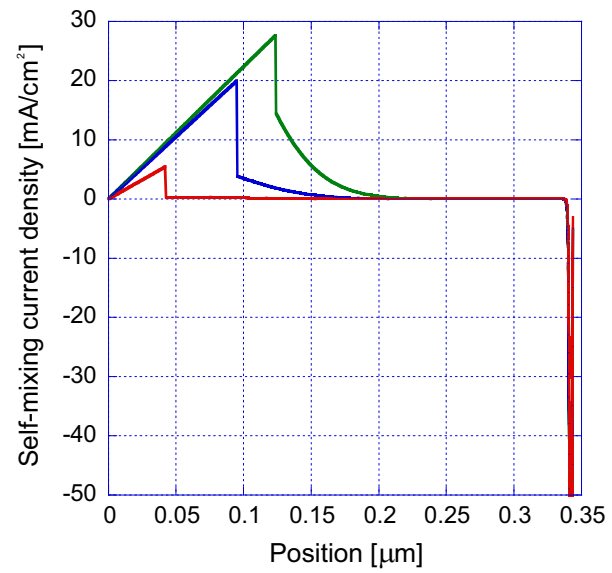

As the frequency increases, the discrimination point between the two solutions moves toward the edges. It results that at 3.2 THZ, the low-doped n-zone is completely covered by $\hat{n}_{A}$ solution, giving rise to a strong reduction of the self-mixing effect. At the highest frequency, the zone close to the anode, with high density of electrons, still presents an $\hat{n}_{B}$ solution, corresponding to a negative contribution to the voltage generated.

The self-mixing terms is the non-homogeneous term in the DC current balance equation. At zero frequency, and in open-circuit condition, the total current in the structure must be 0 . Therefore, the current equation can be written as follows:

$$
J_{\mathrm{DC}}-q n v+q D_{n} \frac{d n}{d x}=0
$$

where $n$ and $v$, from now on, must be intended as the sum of an equilibrium term and of a variation term now due to the self-mixing: $n=n_{0}+\tilde{n}, v=v_{0}+\tilde{v}$. Therefore, Eq. (25) can be rewritten as reported below:

$$
\begin{aligned}
& J_{D C}=q n_{0} \widetilde{v}+q \widetilde{n} v_{0}-q D_{n} \frac{d \widetilde{n}}{d x}= \\
& =-q n_{0} \mu_{n} \widetilde{E}-q \widetilde{n} \mu_{n} E_{0}-q D_{n} \frac{d \widetilde{n}}{d x}
\end{aligned}
$$

$\tilde{E}, \tilde{n}, \frac{d \tilde{n}}{d x}$ are respectively the DC variation of the electric field, electron density, and derivative of the electrons density, introduced in the junction due to the DC self-mixing effect. The self-mixing process gives rise to a variation of the electrostatic potential across the structure. Different contributions to this variation occur from the different portion of the structure itself. These contributions have all the dimension of an electric field, nevertheless, since the different terms in Eq. (26) depicts different physical effects, we chose to define these contributions with an particular symbol, $\tilde{\phi}(y)$. Total potential, $V_{m i x}$, can be obtained by the integration of these contributes

$$
V_{m i x}=\int_{0}^{W} \tilde{\phi}(x) d x
$$

Since $J_{\mathrm{DC}}(x)$ depends on the position inside the structure, the solution of Eq. (26) cannot be directly evaluated. Adopting an "asymptotic" approach, we can assume that only one of the 
three homogeneous terms has a dominant contribution. We can identify the dominant term evaluating the correspondent current contribution. The three solutions are given by the following:

$$
\begin{aligned}
\tilde{E}_{A}(x) & =-\frac{J_{D C(x)}}{q \mu_{n} n_{0}} \\
\tilde{n}_{B}(x) & =-\frac{J_{D C(x)}}{q \mu_{n} E_{0}} \\
\frac{d \tilde{n}_{C}(x)}{d x} & =-\frac{J_{D C(x)}}{q D_{n}}
\end{aligned}
$$

Subscripts specifying the asymptotic solutions, respectively, are indicated as A, B, C. The term $\tilde{E}_{A}$ of Eq. (28) corresponds to a local variation of the electric field, due to the first solution A. The correspondent local differential contribution to the potential, if dominant, is as follows:

$$
\tilde{\phi}_{A}(x)=-\tilde{E}_{A}(x)=\frac{J_{\mathrm{DC}(x)}}{q \mu_{n} n_{0}}
$$

The term in Eq. (29), on the contrary, gives rise to a local variation of the electron density. Since we assume that, in open-circuit condition, all the electrons varying in the junction, for 0 $<x<w_{n}$, are fed by the reserve of electrons contained into non-depleted $\mathrm{n}$ region, there, an equivalent amount of positive charge must be accumulated. We obtain the local potential contribute of the solution B as follows:

$$
\tilde{\phi}_{B}(x)=\frac{J_{D C(x)}}{\varepsilon \mu_{n} E_{0}} x
$$

A homologous solution, $\phi_{B}^{\prime}$, can be written in the zone $w-D^{\prime}>x>w$. Figure 5 sketches the effect of two electron variations $\tilde{n}_{B}$ and $\tilde{n}_{B}{ }^{\prime}$, respectively, in the zone $0<x<w_{n}$ and $w_{n}<x<W$, giving rise to the two contributions to the potential drop $\phi_{B}$ and $\phi_{B}^{\prime}$.

Finally, also the third term in Eq. (26), after a first integration of Eq. (30) leads to the variation of the electron density:

$$
\tilde{n}_{C}(x)=\tilde{n}_{0}+\int_{0}^{x}-\frac{J_{D C(z)}}{q D_{n}} d z
$$

The boundary condition at the border of the depletion layer imposes: $\tilde{n}_{0}=0$. Since we assume all the electrons varying in the junction, for $0<x<w_{n}$ are fed by the reserve of electrons contained into non-depleted $\mathrm{n}$ region, there is an equivalent amount of positive charge is accumulated.

We may obtain also for the third solution the local contribute to the self-mixing potential as follows:

$$
\tilde{\phi}_{C}(x)=-\frac{q}{\varepsilon} \tilde{n}_{C}(x) x
$$

A homologous solution, $\tilde{\phi}_{C}$, can be written in the zone $w-D^{\prime}>x>w$. 


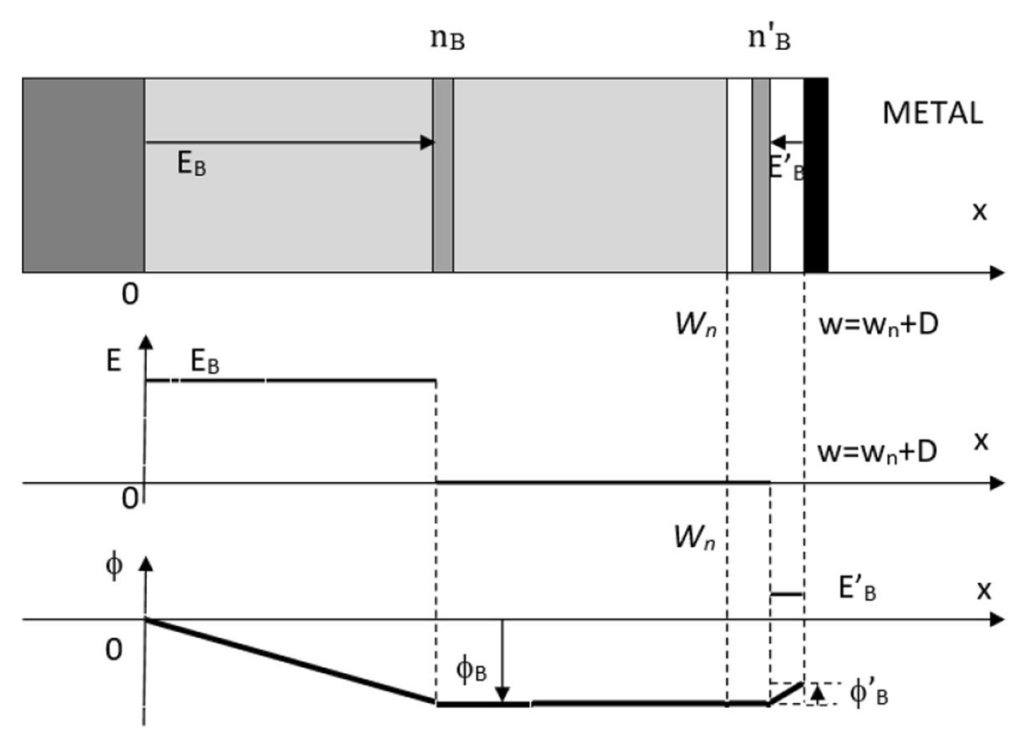

Fig. 5 Sketch of the effect of electrons variations, $n_{\mathrm{B}}$ and $n_{\mathrm{B}}^{\prime}$, respectively, in the zone $0<x<w_{n}$ and $w-D^{\prime}<x$ $<w$, giving rise to the two contributions to the potential drop, $\phi_{\mathrm{B}}$ and $\phi_{\mathrm{B}}^{\prime}$

The three terms formally appear as three current contributes in eq. (26). The sum of the contributions must equate $J_{\mathrm{DC}}$ under the same increment of the potential drop, $\phi(x)$. As asymptotic solution, we choose the one which leads to the contribution with lower increment of the potential drop for a given self-mixing tern, $J_{\mathrm{DC}}$.

The nonlinear term only appears as non-homogenous term; thus, no contributions to DC self-mixing potential comes from charge accumulated at the borders of the depletion layer, $X=$ 0 , and at the metal.

Since the main goal of this paper is the description of the frequency dependence of the selfmixing effect, we choose to avoid the complex analytical solution of the DC potential distribution. A numerical integration of Eq. (27) was thus simply performed. The first result was obtained at open-circuit condition. This condition is often verified in actual measurements, with the measure of DC voltage decoupled from the THz potential applied by the antenna, e.g., this is the standard measurement condition in MOS THz detectors. In this condition, the DC current across the structure was assumed to be null, and the continuity of potential and electric field was imposed along the numerical integration.

Figure 6 reports the behavior of the electrostatic potential generated by the self-mixing process across the double barrier, at the frequencies of $320 \mathrm{GHz}$ and and $3.2 \mathrm{THz}$, respectively. It can be observed how the asymmetry of the barriers generates the overall external potential. Moreover, it is evident as only a portion of the depletion layer contributes to its generation. The calculation of the potential distribution inside the structure was also performed assuming the condition of DC short circuit between anode and cathode (decoupling the DC from the RF boundary condition). The results will be reported in the inset of Fig. 9 for comparison with TCAD simulations. The calculation was performed assuming as the negligibility of the DC current across the structure and imposing the continuity of potential and electric field.

Figure 7 finally reports in log scale the frequency behavior of the double-barrier self-mixing voltage. The low dependence on the frequency can be appreciated. It is compatible with the results of the Monte Carlo simulations reported in [14]. 
Fig. 6 Electric potential generated across the junction by the selfmixing process, at open-circuit condition, at the frequencies of $320 \mathrm{GHz}$ (blue), 1THz (green), and $3.2 \mathrm{THz}$ (red) respectively

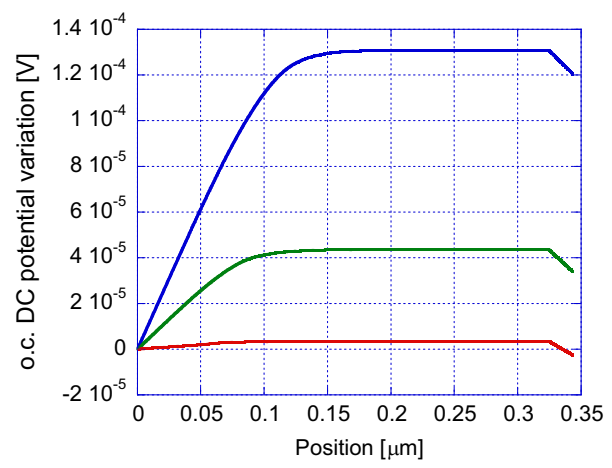

\section{TCAD Simulations}

In order to confirm the results obtained using the proposed model, we performed numerical simulations using Synopsys TCAD of a one-dimensional structure that approximated well the structure used for the model. The $\mathrm{n}^{-}$region was $0.58 \mu \mathrm{m}$ long, with a doping concentration of $5.10^{15} \mathrm{~cm}^{-3}$; the $\mathrm{p}^{+}$region is $50 \mathrm{~nm}$ long, with a doping of $1.10^{18} \mathrm{~cm}^{-3}$; the metal work function is $4.33 \mathrm{eV}$. The structure was slightly longer than the depletion region, in order to avoid interference of the contact with the actual position of the depletion edge.

After simulations, we report in Fig. 8a, b, c, the behavior of the real and imaginary part of the electrons variations in the structure, respectively, obtained at $360 \mathrm{GHz}$ and 1.0 and $3.6 \mathrm{THz}$.

These results are important to confirm the model. In particular, they show the presence of a region of the depleted n-doped region where the variation of the electrons density is in phase with the RF signal. In this region, the real part is much greater than the imaginary part. At the junction, the real part decreases more rapidly than the imaginary part, becoming negligible. Increasing the frequency, the real part of the electron density variations decreases, while the imaginary part presents a maximum result.

Fig. 7 The frequency behavior of the DC self-mixing voltage generated through the structure, with different doping of the $n$ layer

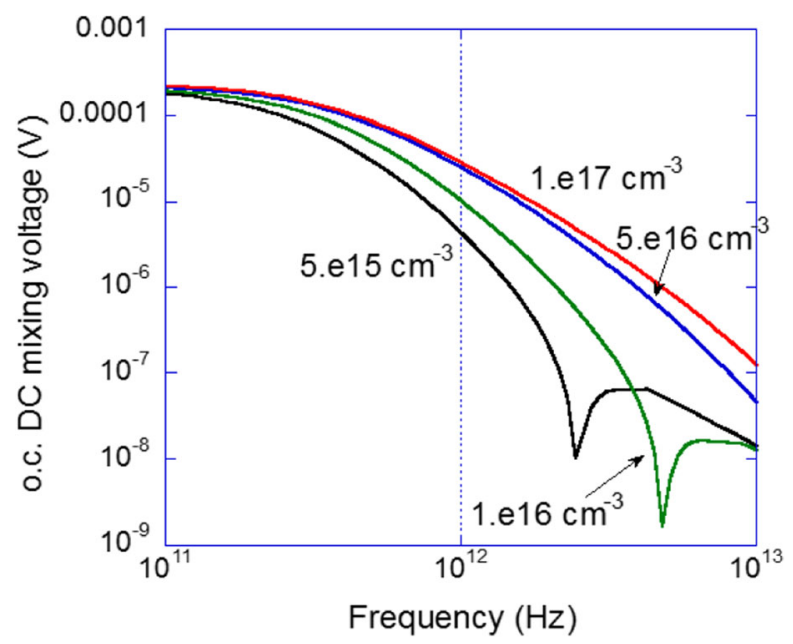



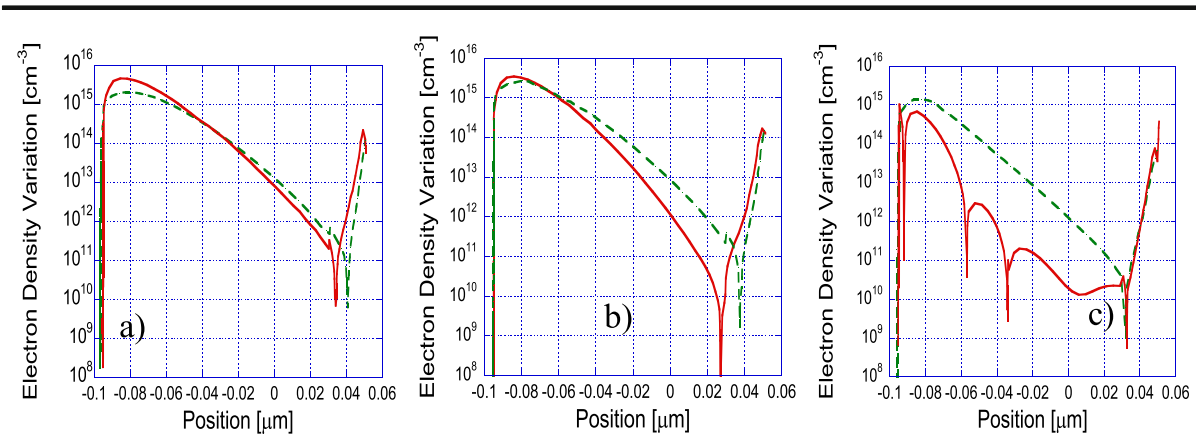

Fig. 8 a-c TCAD simulations of the real (red continuous line) and imaginary (black dashed line) part of the small signal electron density variations, at $320 \mathrm{GHz}$ and 1 and $3.2 \mathrm{THz}$, respectively

Besides the abrupt variations due to the use of the approximation asymptotic adopted, TCAD results and the results obtained using the proposed model (see Fig. 3) are very similar. A straightforward comparison confirms the inversion between the real and imaginary part of the electrons variations within the depletion zone, such as it is confirmed the shrinkage with the frequency of the region where real part overcome the imaginary part. We note that the phase of the variation of the electrons density is a good indicator of the physical effect driving the DC self-mixing. The model indicates that below $1 \mathrm{THz}$, the electron variation (in phase) is due to the predominant effect of diffusion, which overcomes the drift (asymptotic solution B). Indeed, with asymptotic solution B, following Eq. (18), the electron density variations have the same phase of the velocity ones, and the DC mixing term in Eq. (10) is not zero. This term on the contrary is zero for the asymptotic solution A.

The harmonic balance analysis with TCAD was also performed in order to obtain the second order potential distribution. The generation of a second order potential distribution is directly related to the variations induced by the nonlinear self-mixing term. We note that TCAD performs the harmonic balance simulations assuming the condition of short circuit between anode and cathode contacts for all the harmonics, while open-circuit condition at the anode was assumed in the DC analysis of the model, for result shown in Fig. 6. For a correct comparison, the potential distribution was thus calculated using the model imposing DC short circuit at the contacts.

Figure 9 shows the TCAD simulation of the second order potential distribution, generated respectively at the frequency of $320 \mathrm{GHz}$ and 1 and $3.2 \mathrm{THz}$. Results from the model are reported in the inset. The moderate decrease of the pick potential can be observed, with the increasing frequency, and its displacement toward the cathode, corresponding to a reduction of the area active in the self-mixing generation. This behavior, predicted by the model, was effectively described in Fig. 4.

\section{On-Wafer Measurements}

We developed a test structure, which may be obtained with minor changes from a commercial CMOS image sensors (ISs) technology, in order to verify the rectification properties of the double-barrier detector.

Nowadays, CMOS ISs mostly use a pinned photodiode as the photosensitive element of each pixel, constituted by a $\mathrm{p}-\mathrm{n}$ junction which collects photogenerated electrons and the 


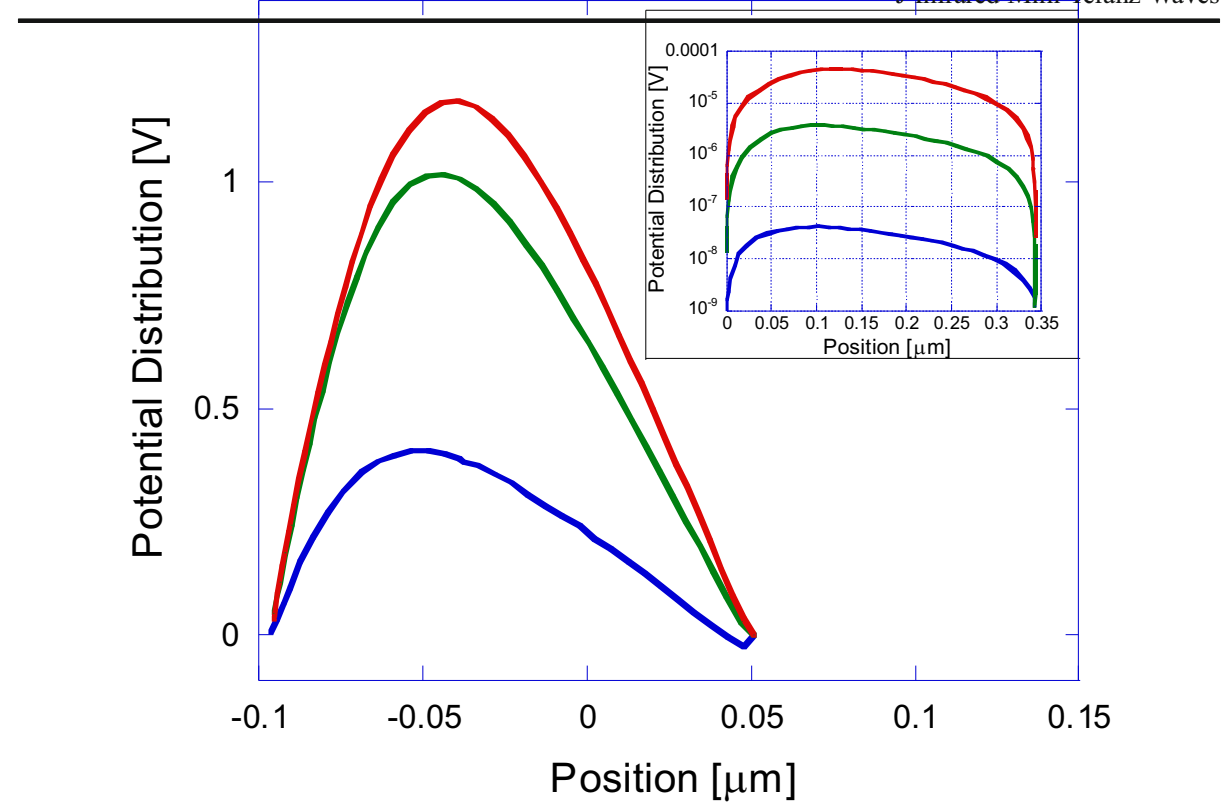

Fig. 9 TCAD simulation of potential distribution inside the structure, respectively, at the frequency of $320 \mathrm{GHz}$ (red), $1 \mathrm{THz}$ (green), $3.2 \mathrm{THz}$ (blue). In the inset: distribution obtained by the model of the potential variation inside the structure calculated under short-circuit condition between anode and cathode, at the frequencies of $320 \mathrm{GHz}$ (red), $1 \mathrm{THz}$ (green), and $3.2 \mathrm{THz}$ (blue) respectively

charge-storage well (SW) capacitance dedicated to the charge integration during the exposure time [9]. A transistor (TX) is connected to the SW to extract the charge accumulated after the integration time and restore the initial condition.

We demonstrated [8] that a modification of a limited region of the surface of the SW, placed in contact with a metal whisker, could result in an efficient rectifying device, with no need to rely on deep submicron technology. The test structure is reported in Fig. 10. The edge of a metal whisker is in contact with the $\mathrm{p}^{+}$-type implanted surface layer, forming a metal-semiconductor junction. The work function of the metal must be such as to ensure a position of the Fermi level similar to those in highly n-doped silicon, e.g., titanium (Ti) with a work function of $4.33 \mathrm{eV}$, a material compatible with CMOS technology, can be used as the terminal portion of the whisker. The $\mathrm{p}^{+} \mathrm{Si}$ layer under the whisker must have a controlled thickness, typically a few tenths of a

Fig. 10 The schematic description of the test structure. Dimensions of RF pads and SW are not to scale

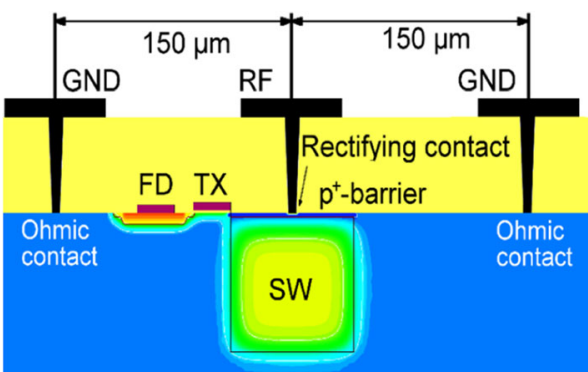

p-type Si wafer 
nanometer, which can be easily implemented by choosing the dose and energy of the ion implantation. The $\mathrm{p}^{+} \mathrm{Si}$ layer and the weakly doped region, ${ }^{-} \mathrm{Si}$, of the $\mathrm{SW}$ form a "second junction." Due to its reduced thickness, the $\mathrm{p}^{+}$Si region becomes almost completely depleted of carriers, giving rise to a reduction of the barrier, to a value of $0.3-0.5 \mathrm{eV}$ with respect to the silicon conduction band inside the SW. The doping concentration of the $\mathrm{p}^{+} \mathrm{Si}$ region can be modified in order to adjust this barrier height. The structure follows the model in Fig. 1.

In an actual receiver, the metal whicker would be the extreme edge of the antenna edge [16]. The antenna coupled to the rectifying device (forming a "rectenna") exposed to the electromagnetic radiation gives rise to voltage variations across the double barrier, the selfmixing produces charge injection into the SW. In the test structure, an RF signal is applied by a radio frequency generator to emulate the excitation coming from the receiving antenna.

At the top metallization level, three pads were realized with dimensions suitable to be contacted by RF microprobes. In particular, Cascade ACP40-GSG-150 microprobes were used during the measurements. The external ground pads were electrically connected to the doped substrate surrounding the SW by means of via holes and ohmic contacts.

Activation of the TX permits the flow of the rectified current toward the pico-ammeter. A bias tee at the input of the VNA ensures the DC continuity toward ground and zeroes the bias voltage from the RF source side.

In the RF, the measurement was performed by using Agilent E8363B VN. The structure was tested at 1 and $40 \mathrm{GHz}$. Figure 11 reports the rectified current measured by a picoammeter versus the RF power applied to the test pad. The TX gate voltage is equal to $2.8 \mathrm{~V}$ for both curves and ensures that TX is in the ON state.

A linear approximation of the curves in Fig. 11 in the non-saturated region can be used to evaluate the system sensitivity, calculating the power necessary to reach the noise level, in particular the relationship between the RF power expressed in decibel-milliwatts, PdBm, and ten times the logarithm of the current, IdBA, as given by

$$
P_{\mathrm{dBm}}=P_{\mathrm{dBm}, 0}+k\left(I_{\mathrm{dBA}}-I_{\mathrm{dBA}, 0}\right),
$$

where $P_{\mathrm{dBm}, 0}, I_{\mathrm{dBA}, 0}$, and the coefficient $k$ can be calculated from geometrical regression of the measured data. This approach makes it possible to account for eventual nonlinearities in the

Fig. 11 Measurement of current variations in the time integration regime at $1 \mathrm{GHz}$ (squares) and $40 \mathrm{GHz}$ (circles), from [8]. Dashed lines are the fitting in the nonsaturated zone

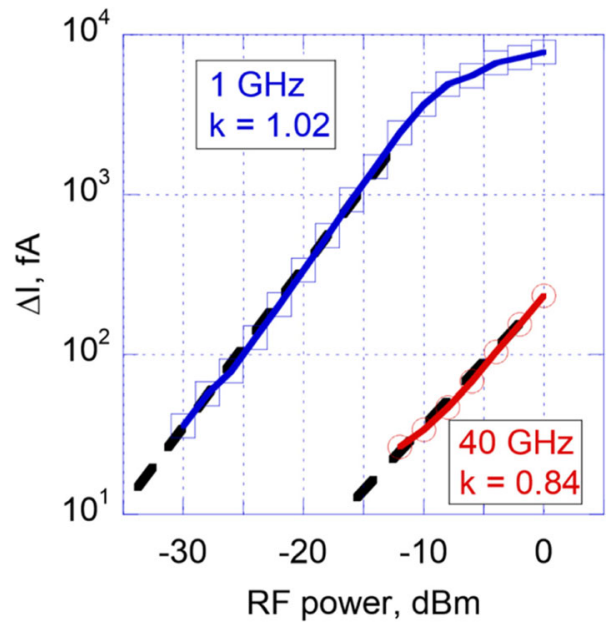


rectification. From the measurement at $1 \mathrm{GHz}$, we obtained $k=1.02$, while at $40 \mathrm{GHz}$ the coefficient resulted in $k=0.84$.

These results can now have a new interpretation in term of the self-mixing process. The different values of $k$ indeed can be related to the generation of the DC potential distribution inside the structure, as reported in Fig. 10. This potential, which is higher at the lower frequencies, partially drops the barriers in the short-circuit conditions of the measurement. The drop is proportional to the applied RF power thus resulting in a change of the slope of the log-log plot.

We believe that the one just presented can represent a good experimental demonstration of the model, even if the frequency behavior still deserves a specific check.

\section{Conclusions}

We presented a new model of the self-mixing process in the double-barrier device. The doublebarrier rectifier is a new structure of $\mathrm{THz}$ detector recently proposed, which can be realized by reuse of CMOS image sensor technology. The model is based on the hydrodynamic semiconductor equations, solved in the small signal approximation. The detector was assumed in the zero bias condition, as expected in a detector in order to reduce the junction noise. The model effectively describes the frequency dependence of the detection capability of the structure, which is not related to the damping effect of the parasitic capacitances, rather to the nonlinear effect of the carrier's dynamic within the depletion layer, the self-mixing process. The model thus substantially improves the analytical models of the self-mixing available in literature, mainly based on lamped equivalent circuits.

In particular, the model depicts the mechanisms of the THz modulation of the charge in the depleted regions of the structure. The behaviors of the model were confirmed by TCAD simulations.

Comparison with literature results on the Schottky diode frequency response, the and Monte Carlo simulations indicate that the model could be extended also to other $\mathrm{THz}$ detectors structures.

The model furnishes new insight on former characterizations of the double-barrier structure.

Acknowledgments The authors would like to thank Aptina for allowing the realization of the test structure in the state-of-art CMOS IS technology.

\section{References}

1. Al Hadi, R., Sherry, H., Grzyb, J., Baktash, N., Zhao, Y., Öjefors, E., Pfeiffer, U., Microwave Symposium Digest (MTT), 2011 I.E. MTT-S International (pp. 1-4), (2011).

2. Lisauskas, A., Boppel, S., Krozer, V., \& Roskos, H. G., Sensors, 2011 I.E. (pp. 55-58). (2011).

3. Grzyb, J., Sherry, H., Zhao, Y., Al Hadi, R., Cathelin, A., Kaiser, A., \& Pfeiffer, U., SPIE Defense, Security, and Sensing (pp. 83620C-83620C). International Society for Optics and Photonics (2012).

4. Corcos, D., Brouk, I., Malits, M., Svetlitza, A., Stolyarova, S., Abramovich, A., Nemirovsky, Y., Microwaves, Communications Antennas and Electronics Systems (COMCAS), 2011 I.E. International Conference on (pp. 1-4). (2011).

5. Dyakonov, M., \& Shur, M., IEEE Transactions on Electron Devices, 43(3), 380-387 (1996).

6. Schuster, F., Coquillat, D., Videlier, H., Sakowicz, M., Teppe, F., Dussopt, L., Knap, W., Optics Express, 19(8), 7827-7832 (2011).

7. Zhu, Y., Ji, X., Liao, Y., Wu, F., \& Yan, F., Solid-State and Integrated Circuit Technology (ICSICT), 2014 12th IEEE International Conference on (pp. 1-3). (2014). 
8. Varlamava, V., De Amicis, G., Del Monte, A., Perticaroli, S., Rao, R., Palma, F., Journal of Infrared, Millimeter, and Terahertz Waves, 37(8), 737-752 (2016).

9. Fossum, E. R., \& Hondongwa, D. B., IEEE J. Electron Devices Soc., 2(3), 33-43 (2014).

10. Siles, J. V., \& Grajal, J., IEEE Transactions on Microwave Theory and Techniques, 58(7), 1933-1942 (2010).

11. Shashkin, V. I., \& Vostokov, N. V., IEEE Journal of the Electron Devices Society, 1(3), 76-82 (2013).

12. Champlin, K. S., \& Eisenstein, G., IEEE Transactions on Microwave Theory and Techniques, 26(1), 31-34 (1978).

13. Han, R., Zhang, Y., Coquillat, D., Videlier, H., Knap, W., \& Brown, E., IEEE Journal of Solid-State Circuits, 46(11), 2602-2612 (2011).

14. Shiktorov, P., Starikov, E., Gruzinskis, V., Pérez, S., González, T., Reggiani, L, Vaissiere, J. C., IEEE Electron Device Letters, 25(1), 1-3.

15. Palma, F., Das, P. K. (1987). Acoustoelectric interaction in layered semiconductor. IEEE transactions on ultrasonics, ferroelectrics, and frequency control, 34(3), 376-382 (2004).

16. Varlamava, V., Palma, F., Nenzi, P., Balucani, M., IEEE Transactions on Terahertz Science and Technology, 4(3), 360-367 (2014). 